\title{
S Ori J053825.4-024241: a classical T Tauri-like object at the substellar boundary ${ }^{\star}$
}

\author{
J. A. Caballero ${ }^{1}$, E. L. Martín ${ }^{1,2}$, M. R. Zapatero Osorio ${ }^{3}$, V. J. S. Béjar ${ }^{4}$, \\ R. Rebolo ${ }^{1,5}$, Ya. Pavlenko ${ }^{6,7}$, and R. Wainscoat ${ }^{8}$
}

\author{
${ }^{1}$ Instituto de Astrofísica de Canarias, 38205 La Laguna, Tenerife, Spain \\ e-mail: zvezda@ll.iac.es \\ 2 University of Central Florida, Dept. of Physics, PO Box 162385, Orlando, FL 32816-2385, USA \\ 3 LAEFF-INTA, PO Box 50727, 28080, Madrid, Spain \\ 4 Proyecto Gran Telescopio Canarias, Instituto de Astrofísica de Canarias \\ 5 Consejo Superior de Investigaciones Científicas, Spain \\ ${ }^{6}$ Centre for Astrophysics Research, University of Hertfordshire, College Lane, Hatfield, Hertfordshire AL10 9AB, UK \\ 7 Main Astronomical Observatory of Academy of Sciences of Ukraine, Golosiiv Woods, Kyiv-127, 03680, Ukraine \\ 8 Institute for Astronomy, University of Hawai'i, 2680 Woodlawn Drive, Honolulu, HI 96822, USA
}

Received 18 May 2005 / Accepted 24 August 2005

\section{ABSTRACT}

We present a spectrophotometric analysis of S Ori J053825.4-024241, a candidate member close to the substellar boundary of the young $(1-8 \mathrm{Myr})$, nearby $(\sim 360 \mathrm{pc}) \sigma$ Orionis star cluster. Our optical and near-infrared photometry and low-resolution spectroscopy indicate that S Ori J053825.4-024241 is a likely cluster member with a mass estimated from evolutionary models at $0.06_{-0.02}^{+0.07} M_{\odot}$, which makes the object a probable brown dwarf. The radial velocity of S Ori J053825.4-024241 is similar to the cluster systemic velocity. This target, which we have classified as an M $6.0 \pm 1.0$ low-gravity object, shows excess emission in the near-infrared and anomalously strong photometric variability for its type (from the blue to the $J$ band), suggesting the presence of a surrounding disc. The optical spectroscopic observations show a continuum excess at short wavelengths and a persistent and resolved $\mathrm{H} \alpha$ emission (pseudo-equivalent width of $\sim-250 \AA$ ) in addition to the presence of other forbidden and permitted emission lines, which we interpret as indicating accretion from the disc and possibly mass loss. We conclude that despite the low mass of S Ori J053825.4-024241, this object exhibits some of the properties typical of active classical T Tauri stars.

Key words. stars: low mass, brown dwarfs - accretion, accretion discs - stars: variables: general Galaxy: open clusters and associations: individuals: $\sigma$ Orionis

\section{Introduction}

$\mathrm{T}$ Tauri stars are the bridge between new-born embedded protostars and zero age main sequence stars. The most widely accepted scenario proposed to explain all their features is the magnetospheric accretion of mass infalling from a surrounding circumstellar disc. Bertout et al. (1988), Appenzeller \& Mundt (1989) and Königl (1991) have reviewed the properties of $\mathrm{T}$ Tauri stars and their discs. The existence of a T Tauri-like phase in brown dwarfs, objects below the hydrogen-burning mass limit $\left(\sim 0.072 M_{\odot}\right.$ for solar metallicity) has been suggested in the past few years. See, for example, Muzerolle et al. (2003), Barrado y Navascués \& Martín (2003), Natta et al. (2004), or the most recent papers by Mohanty et al. (2005) and Furlan et al. (2005) and references therein.

^ Acknowledgments are only available in electronic form at http://www . edpsciences.org
S Ori J053825.4-024241 was firstly identified as a photometric substellar candidate of the young $\sigma$ Orionis cluster (Béjar et al. 2004). Hence, it is probably 1-8 Myr old and is located at a distance of about 360 pc (see Béjar et al. 2001 for further details on the cluster properties). S Ori J053825.4-024241 exhibits considerable excess emission in the $K_{\mathrm{s}}$-band, whereas the $R-I$ colour appears to be slightly bluer than the colour of other cluster members of similar magnitude. Caballero et al. (2004) found S Ori J053825.4-024241 to be one of the most photometric variable sources of its spectral type, with variations as high as $0.36 \mathrm{mag}$ in the $I$ band over a few hours.

In this paper we present optical spectroscopy that confirms the membership of S Ori J053825.4-024241 in $\sigma$ Orionis at the borderline between stars and brown dwarfs. Both photometric and spectroscopic data suggest that this object is undergoing active mass accretion. The presence of circumstellar discs around low-mass stars and brown dwarfs in the $\sigma$ Orionis cluster has been inferred by several authors (e.g., 


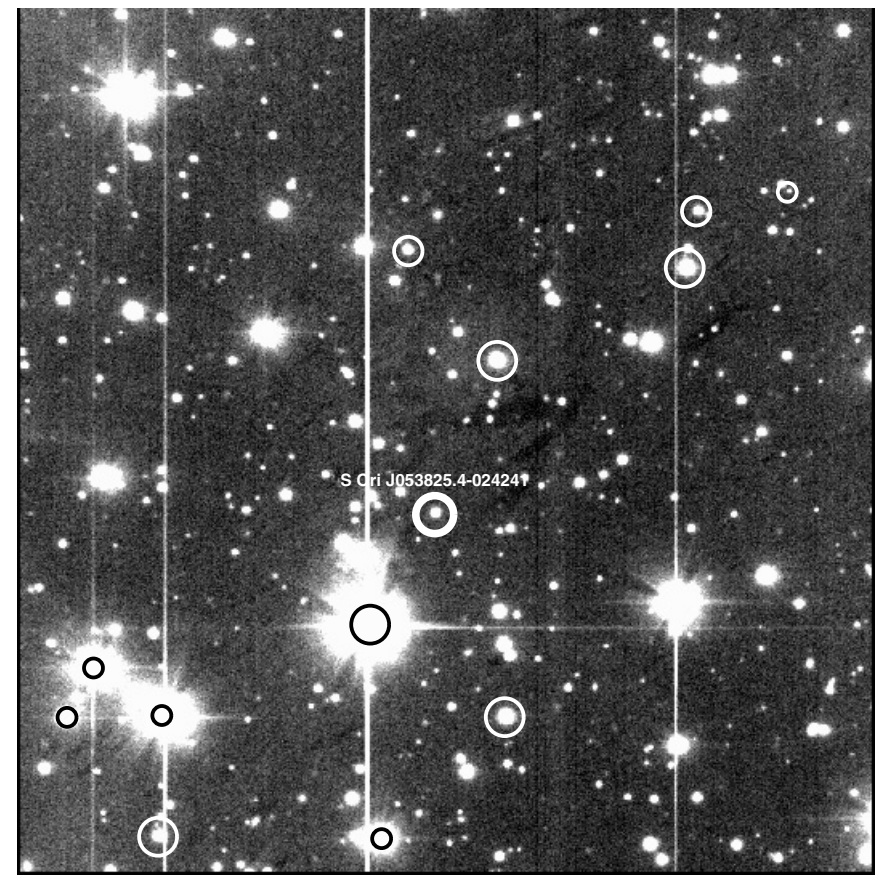

Fig. 1. Finder chart of S Ori J053825.4-024241 (labelled) taken in the I-band with the IAC-80 telescope. Other stellar and substellar members of the $\sigma$ Orionis cluster are indicated with circles. Field of view is $6.5 \times 6.5 \mathrm{arcmin}^{2}$. North is at the top and east to the left.

Oliveira et al. 2004 and Scholz \& Eislöffel 2004). However, the particular peculiarities of S Ori J053825.4-024241 deserve intensive study.

\section{Observations and analysis}

\subsection{Optical spectroscopy}

We have obtained low-resolution spectra of S Ori J053825.4024241 using the $2.56 \mathrm{~m}$ Nordic Optical Telescope (NOT, Observatorio del Roque los Muchachos, Spain) and the $10 \mathrm{~m}$ Keck I Telescope (W. M. Keck Observatory, Hawai'i). The finder chart of this object is depicted in Fig. 1, and Table 1 provides the log of the spectroscopic observations. Raw data were reduced using tasks within the IRAF environment. Reduction steps included bias subtraction, flat-fielding, optimal extraction, wavelength calibration, and correction for instrumental response and telluric contribution.

We used the Andalucía Faint Object Spectrograph and Camera (ALFOSC) instrument attached to the Cassegrain focus of the NOT to obtain a spectrum of S Ori J053825.4-024241 $(R \sim 600)$. See Table 1 for details on the observations and the instrumental configuration. We note the strong fringing redwards of $7600 \AA$, and the low number of photons bluewards of $\sim 6000 \AA$. Observations were hampered by poor weather conditions (cirrus and high relative humidity).

We also obtained spectra of three additional $\sigma$ Orionis candidate members of similar brightness on the same night $(I \sim$ 17 mag, namely S Ori J053954.3-023719, S Ori J053838.6024157 and S Ori J053826.1-024041). These were found in the photometric searches of Béjar et al. (2004) and Caballero et al. (2004). These authors reported on the short-scale variable
Table 1. Log of the spectroscopic observations.

\begin{tabular}{lcc}
\hline \hline & NOT & Keck \\
\hline Date & 2003 Dec. 26 & 2004 Jan. 23 \\
MJD & 52999.93 & 53027.31 \\
No. of spectra & 3 & 2 \\
Exposure time & $3 \times 900 \mathrm{~s}$ & $1200+390 \mathrm{~s}$ \\
Telescope & NOT & Keck I \\
Instrument & ALFOSC & LRIS \\
Detector & E2V $2 \mathrm{k} \times 2 \mathrm{k} 42-40$ & Tektronics $2 \mathrm{k} \times 2 \mathrm{k}$ \\
Grism & $\# 5$ & \#4 \\
Blocking filter & GG475 & GG495 \\
$\lambda$ coverage & $5300-9600 \AA$ & $5600-8100 \AA$ \\
Pixel size & 0.19 arcsec & $0.213 \operatorname{arcsec}^{-1}$ \\
Slit width & 1.3 arcsec & $1.0 \operatorname{arcsec}^{-1}$ \\
Dispersion & $3.1 \AA$ pixel & $1.25 \AA$ pixel $^{-1}$ \\
Resolution & $12 \AA$ & $4.5 \AA$ \\
\hline
\end{tabular}

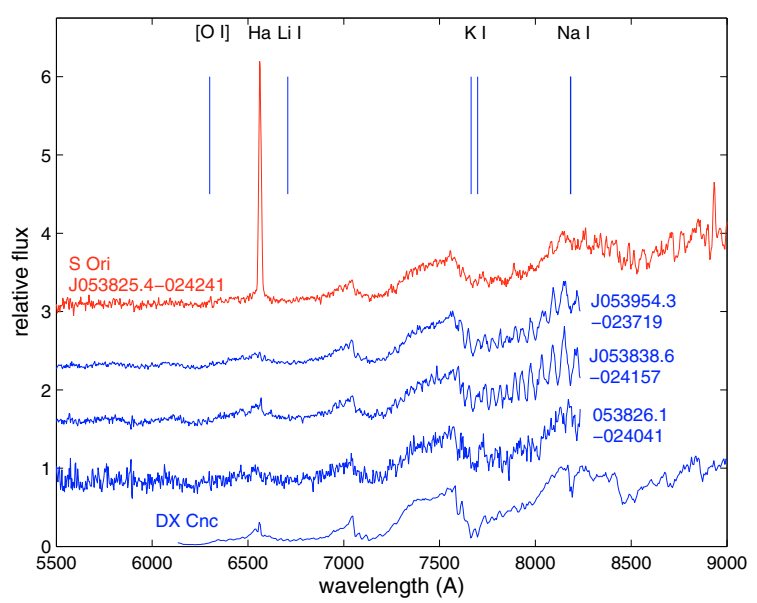

Fig. 2. Low-resolution NOT spectra of $\sigma$ Orionis candidates. The top spectrum corresponds to S Ori J053825.4-024241. For the other objects, the data redwards of $8200 \AA$ are not displayed because of their very poor signal-to-noise ratio. The spectrum of the field M6.5V-type dwarf DX Cnc (Martín et al. 1996), which has been degraded to the same resolution as the NOT data, is also displayed for comparison. Spectra have been shifted in the vertical axis for clarity. Several atomic features are labelled. Coloured versions of all the figures can be found in the electronic version.

nature of S Ori J053826.1-024041. Barrado y Navascués et al. (2003) classified S Ori J053826.1-024041 as an M8-dwarf member of the cluster. Exposure times were $3 \times 900 \mathrm{~s}$ for S Ori J053825.4-024241, and $1000 \mathrm{~s}$ for each of the other three objects. Figure 2 shows the NOT spectra, which were corrected for telluric lines by observing featureless stars.

Spectral types were derived by measuring the pseudocontinuum indices (PC) of Martín et al. (1996) and by direct comparison to well-known late-type spectroscopic standard stars. We provide our measurements in Table 2. We note that the PC1 index is affected by the broad $\mathrm{H} \alpha$ emission of S Ori J053825.4-024241, and that the PC4 index suffers from the strong fringing of our data. $\mathrm{PC} 2$ and $\mathrm{PC} 3$ remain, however, 
Table 2. I-band magnitude, $\mathrm{H} \alpha$ emission, pseudocontinuum indices and spectral types from the NOT data.

\begin{tabular}{|c|c|c|c|c|c|c|c|}
\hline Name & $\begin{array}{c}I^{a} \\
(\mathrm{mag})\end{array}$ & $\begin{array}{c}p E W(\mathrm{H} \alpha) \\
(\AA)\end{array}$ & $\mathrm{PC} 1$ & PC2 & PC3 & $\mathrm{PC} 4$ & Spectral type \\
\hline S Ori J053825.4-024241 & $16.86 \pm 0.06$ & $-230 \pm 70$ & $1.2 \pm 0.6$ & $1.85 \pm 0.17$ & $1.36 \pm 0.13$ & $1.7 \pm 0.3$ & $\mathrm{M} 6.0 \pm 1.0$ \\
\hline S Ori J053954.3-023719 & $17.03 \pm 0.04$ & $-5 \pm 1$ & $1.8 \pm 0.2$ & $2.0 \pm 0.2$ & $1.34 \pm 0.18$ & $1.64 \pm 0.12$ & $\mathrm{M} 6.0 \pm 1.0$ \\
\hline S Ori J053838.6-024157 & $16.52 \pm 0.07$ & $-6 \pm 1$ & $1.39 \pm 0.14$ & $1.75 \pm 0.18$ & $1.3 \pm 0.3$ & $1.6 \pm 0.2$ & M5.5 \pm 1.0 \\
\hline S Ori J053826.1-024041 & $16.87 \pm 0.06$ & {$[-2,+2]^{b}$} & $1.4 \pm 0.4$ & $2.2 \pm 0.5$ & $1.19 \pm 0.18$ & $1.5 \pm 0.3$ & $\mathrm{M} 5.0 \pm 2.0^{c}$ \\
\hline
\end{tabular}

${ }^{a}$ From Béjar et al. (2004).

${ }^{b} p E W(\mathrm{H} \alpha)=-4 \pm 2 \AA$ (from Barrado y Navascués et al. 2003).

${ }^{c}$ M6.0 spectral type matches better to the spectroscopic cluster sequence. It is an M8-type binary dwarf according to Barrado y Navascués et al. (2003).

as reliable indicators of the spectral type. In case of strong veiling due to a blue continuum excess, the PC indices may provide spectral types that are slightly earlier than the real ones. The polynomial fitting to the PC index-spectral type relations given in Martín et al. (1996) yields spectral types between M5 and M6 for the four objects in our work. In particular, we have determined that the spectral type of S Ori J053825.4-024241, the main target of our paper, is M6 with an uncertainty of one subtype.

As seen from Fig. 2, the most prominent spectral feature of S Ori J053825.4-024241 is $\mathrm{H} \alpha$, which is seen in strong emission. We measured a pseudoequivalent width ( $p E W$, equivalent width with respect to the observed continuum) of $-230 \pm 70 \AA$ on the NOT spectrum, which makes S Ori J053825.4-024241 one of the largest $\mathrm{H} \alpha$ emitters among $\sigma$ Orionis late-type members. The other three objects do not display such intense $\mathrm{H} \alpha$ lines (see Table 2). The forbidden line of [OI] $\lambda 6300.3$ is also detected in emission with a moderate strength in the NOT spectrum of S Ori J053825.4-024241. Regarding absorption features, all $\sigma$ Orionis objects show rather weak K I $\lambda \lambda 7664.9,7699.0$ and $\mathrm{Na}$ I $\lambda \lambda 8183.3,8148.8$ lines as compared to field dwarfs of similar types (see Fig. 2). Although the doublets are blended at the low resolution of the NOT data, they should appear with $p E W$ s of $20 \AA$ and $6 \AA$ in $\sim \mathrm{M} 6 \mathrm{~V}$-type spectra, respectively. However, we can only impose upper limits that are significantly smaller. This is indicative of low-gravity atmospheres (Martín et al. 1996; Luhman et al. 1997) or of the presence of a strong continuum excess, both supporting the young age of the objects and their membership of the $\sigma$ Orionis cluster. We note that our $p E W$ s of the NaI $\lambda \lambda 8183.3,8148.8$ doublet are in agreement with the measurements of Kenyon et al. (2005) obtained for various M5-6-type $\sigma$ Orionis members. Another excellent indicator of youth is the presence of Li I $\lambda 6707.8$ absorption. The low resolution and moderate signal-to-noise ratio of the NOT spectra do not allow a clear detection of this feature. Table 3 provides our measurements of S Ori J053825.4-024241.

To check the stability of the $\mathrm{H} \alpha$ emission of S Ori J053825.4-024241, we collected two optical spectra with a higher resolution $(R \sim 1700)$ of $1200 \mathrm{~s}$ and $390 \mathrm{~s}$ about one month later than the NOT data (see Table 1). The Low Resolution Imaging Spectrograph (LRIS) was used at the Keck I Telescope. The Keck spectra were
Table 3. S Ori J053825.4-024241: NOT data.

\begin{tabular}{cr}
\hline \hline Line & $p E W(\AA)$ \\
\hline [O I $] \lambda 6300.3$ & $-2.5 \pm 1.0$ \\
H $\alpha \lambda 6562.8$ & $-230 \pm 70$ \\
Li I $\lambda 6707.8$ & $<+0.7$ \\
K I $\lambda 7664.9^{a}$ & $<+2.0$ \\
K I $\lambda 7699.0^{a}$ & $<+2.0$ \\
Na I $\lambda 8183.3^{a}$ & $<+2.0$ \\
Na I $\lambda 8184.8^{a}$ & $<+2.0$ \\
\hline & Line measurements affected by fringing.
\end{tabular}

wavelength-calibrated using emission sky lines (Osterbrock et al. 1996), which were observed along with the target. The root-mean-square of the polynomial fit to the calibration is typically $0.28 \AA$. The data were not corrected for instrumental response or terrestrial contribution because no flux standard star was observed owing to very poor weather conditions. Of the two spectra, the first exposure shows better signal-to-noise ratio. This spectrum is depicted in Fig. 3. The second spectrum is of very poor quality. However, $\mathrm{H} \alpha$ is clearly detected in strong emission, as it is in the first spectrum. The Keck data confirm the persistent emission of S Ori J053825.4-024241. The profile of the observed $\mathrm{H} \alpha$ emission is shown in Fig. 4. We have plotted relative fluxes as a function of the velocity shift from line center and scaled to the peak $\mathrm{H} \alpha$ flux of the object. $\mathrm{H} \alpha$ appears rather symmetric and broad. It is resolved as compared to telluric emission lines at similar wavelengths.

Besides $\mathrm{H} \alpha$, we have also detected emission from $\mathrm{He} I\left(\mathrm{D}_{3}\right) \lambda 5875.8$ and $[\mathrm{OI}] \lambda 6300.3$ (Fig. 4). No significant variations are seen between the two consecutive Keck spectra. Pseudo-equivalent widths measured from the best-quality Keck spectrum are given in Table 4. As inferred from the strength of the emission lines (Tables 3 and 4), the activity level of S Ori J053825.4-024241 remains approximately constant between the two epochs of observation. We also provide in Table 4 the $p E W$ s of several Na I, K I, Li I and Ca I lines. Note that the KI doublet is severely affected by telluric absorption. We remark that only upper limits can be set for Li I $\lambda 6707.8$ (Fig. 3) and the Na I $\lambda \lambda 5890.0,5895.9$ doublet. The Na I and KI lines (Fig. 3) are detected much weaker than expected for high-gravity dwarfs of similar spectral type. 


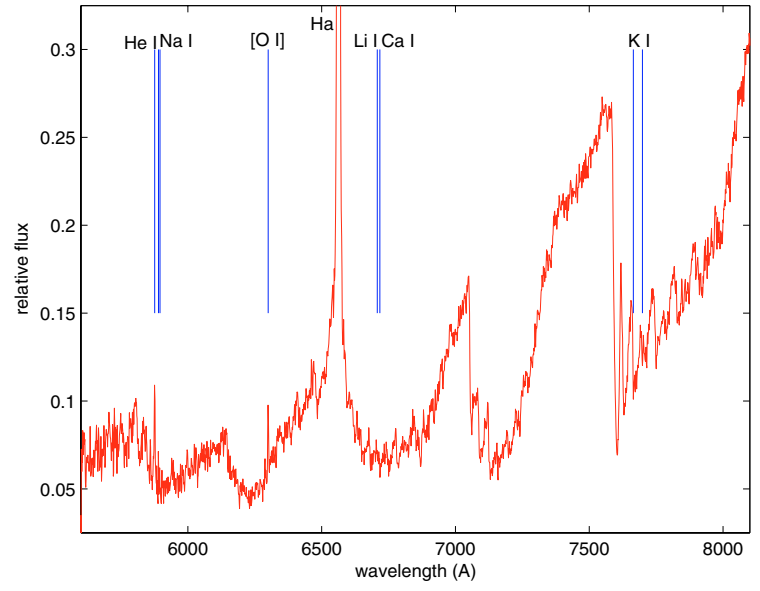

Fig. 3. Keck spectrum of S Ori J053825.4-024241. No correction for instrumental response and telluric contribution has been applied.
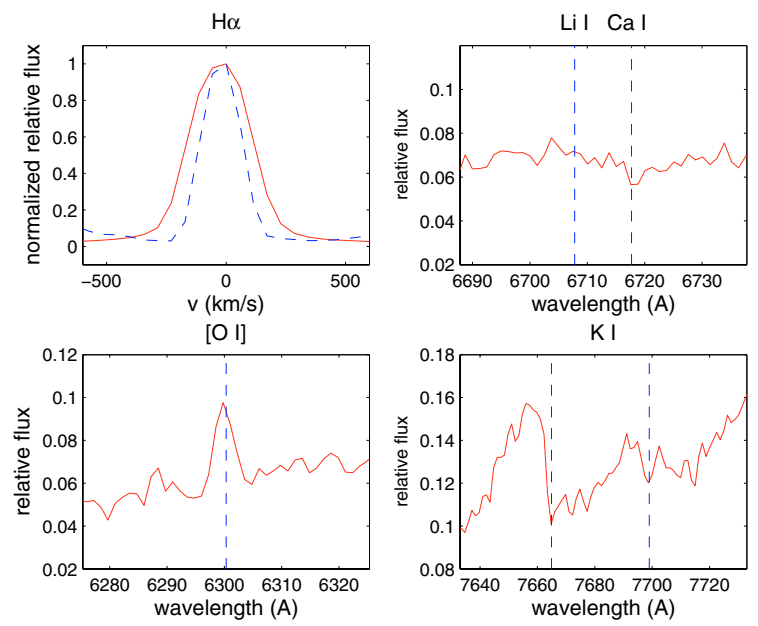

Fig. 4. Details of the Keck spectrum of S Ori J053825.4-024241. (Top left) The $\mathrm{H} \alpha$ profile (full line) normalized to the peak and compared to one sky emission line (dashed line) at around a similar wavelength. (Top right) Lithium non-detection with an upper limit on the $p E W$ of $+0.3 \AA$. (Bottom left) Emission from [O I]. (Bottom right) Spectral region around the K I $\lambda \lambda 7664.9,7699.0$ doublet.

The heliocentric radial velocity of S Ori J053825.4024241 was computed via Fourier cross-correlation of the object best Keck spectrum with the field standard star V1298 Aql (vB 10, M8 V, $v_{\mathrm{h}}=35.3 \pm 1.5 \mathrm{~km} \mathrm{~s}^{-1}$, Tinney \& Reid 1998). This template star was also observed with the Keck Telescope and similar instrumentation on a previous run. We took special care in cross-correlating spectral windows (6620-6840 , and 7000-7260 $)$ that are not affected by telluric absorptions and that contain many photospheric lines (particularly TiO). Additionally, we considered parts of the spectrum free of emission lines. We derived $v_{\mathrm{h}}=+32 \pm 13 \mathrm{~km} \mathrm{~s}^{-1}$ for S Ori J053825.4-024241, which is in full agreement within the error bars with the mean heliocentric radial velocity of $\sigma$ Orionis members (Walter et al. 1998; Zapatero Osorio et al. 2002a). Velocities were also computed for each emission line of the spectrum by measuring line centroids. Observed velocities were corrected for the rotation of the Earth, the motion of the Earth about the Earth-Moon barycentre, and the orbit of the
Table 4. S Ori J053825.4-024241: Keck data.

\begin{tabular}{|c|c|c|}
\hline Line & $p E W(\AA)$ & $v_{\mathrm{h}}\left(\mathrm{km} \mathrm{s}^{-1}\right)$ \\
\hline He I $\lambda 5875.8$ & $-3.5 \pm 1.0$ & $+29 \pm 13^{a}$ \\
\hline $\mathrm{Na}$ I $\lambda 5890.0$ & $<+0.1$ & - \\
\hline $\mathrm{Na}$ I $\lambda 5895.9$ & $<+0.1$ & - \\
\hline [O I] $\lambda 6300.3$ & $-2.1 \pm 0.5$ & $+7 \pm 13^{a}$ \\
\hline Н $\alpha \lambda 6562.8$ & $-260 \pm 30$ & $+23 \pm 13^{a}$ \\
\hline$[\mathrm{N}$ II] $\lambda 6583.5$ & {$[-0.2,+0.2]$} & - \\
\hline Li I $\lambda 6707.8$ & $<+0.3$ & - \\
\hline Са I $\lambda 6717.7$ & $+0.5 \pm 0.1$ & $+32 \pm 13^{b}$ \\
\hline K I $\lambda 7699.0$ & $+0.5 \pm 0.1$ & $+32 \pm 13^{b}$ \\
\hline
\end{tabular}

${ }^{a}$ Velocity obtained from the line centroid.

${ }^{b}$ Velocity obtained from the cross-correlation technique.

barycentre about the Sun to yield heliocentric radial velocities, which are listed in Table 4 . We note that the forbidden emission due to $[\mathrm{O} \mathrm{I}]$ appears blue-shifted in velocity.

\subsection{Optical photometry}

We have also conducted multiwavelength optical and nearinfrared broad-band observations of S Ori J053825.4-024241 using the $R, I, J$ and $H$ filters and in white light. These data, which we have added to previous measurements available in the literature (2MASS photometry, Béjar et al. 2004; and Caballero et al. 2004, 2005), have been employed to monitor photometric variability on different timescales. Broadband photometry has been obtained using the following telescopes: the $0.8 \mathrm{~m}$ IAC- 80 , the $1 \mathrm{~m}$ Optical Ground Station (OGS) and the $1.52 \mathrm{~m}$ Telescopio Carlos Sánchez (TCS) at the Teide Observatory, the $2.5 \mathrm{~m}$ Isaac Newton Telescope (INT) at Roque de los Muchachos Observatory, the $3.5 \mathrm{~m}$ Calar Alto Telescope at Calar Alto Observatory, and the $8 \mathrm{~m}$ Very Large Telescope UT1 (Antu) at Paranal Observatory. In Table 5 we provide the $\log$ of the observations. Listed are the Modified Julian Date of the beginning of the observations, total number of exposures and integration time per exposure, temporal coverage per observing date, and filter and instrument used.

Raw frames were reduced within IRAF using standard techniques in the optical and near-infrared wavelengths. We have conducted a careful differential photometric analysis of all the data in the same manner as in Caballero et al. (2004). We refer to this paper for a description of the analysis.

\subsubsection{I-band differential photometry}

Using the Thomson $1024 \times 1024 \mathrm{CCD}$ camera $(0.4325$ arcsec pixel $^{-1}$ ) at the IAC-80 telescope, we have carried out time-series differential $I$-band photometry in a region of $6.5 \times$ $6.5 \operatorname{arcmin}^{2}$ around S Ori J053825.4-024241. Our target was monitored during six nights. On two of these nights, it was followed for more than two hours. A total of 45 images have been used to build the IAC-80 I-band light curve of the object, which is shown in Fig. 5. The minimum variation amplitude detectable is $0.025 \mathrm{mag}$. The seeing ranged from 1.2 to 2.0 arcsec 
Table 5. Log of the photometric observations.

\begin{tabular}{|c|c|c|c|c|c|c|c|}
\hline Observing date & $\begin{array}{l}\text { Start } \\
\text { MJD }\end{array}$ & $\begin{array}{c}\text { Exposure } \\
\text { time (s) }\end{array}$ & $\begin{array}{l}\Delta t \\
(\mathrm{~h})\end{array}$ & Telescope & Instrument & Filter & Reference \\
\hline 1998 Jan. 22 & 50835.929 & $1 \times 1800$ & $<1$ & IAC-80 & $\mathrm{CCD}$ & $I$ & Béjar et al. (2004) \\
\hline 1998 Jan. 22 & 50835.983 & $1 \times 1800$ & $<1$ & IAC-80 & $\mathrm{CCD}$ & $R$ & Béjar et al. (2004) \\
\hline 1999 Jan. 22 & 51201.047 & $1 \times 720$ & $<1$ & TCS & CAIN-2 & $J$ & this paper \\
\hline 1999 Jan. 24 & 51202.870 & $1 \times 720$ & $<1$ & TCS & CAIN-2 & $J$ & Béjar et al. (2004) \\
\hline 2000 Dec. 30 & 51909.075 & $5 \times 1500$ & 1.4 & INT & WFC & $I$ & Caballero et al. (2004) \\
\hline 2000 Dec. 31 & 51909.901 & $10 \times 1500$ & 4.7 & INT & WFC & $I$ & Caballero et al. (2004) \\
\hline 2001 Jan. 01 & 51911.055 & $6 \times 1500$ & 5.2 & INT & WFC & $I$ & Caballero et al. (2004) \\
\hline 2001 Dec. 10 & 52254.295 & $1 \times 1920$ & $<1$ & Antu & ISAAC & $J$ & Caballero et al. (2005) \\
\hline 2003 Jan 12 & 52647.414 & $12 \times 1200$ & 4.2 & INT & WFC & $I$ & Caballero et al. (2004) \\
\hline 2003 Oct. 19 & 52932.023 & $1 \times 3600$ & $<1$ & $3.5 \mathrm{~m} \mathrm{CA}$ & $\mathrm{o} 2 \mathrm{~K}$ & $H$ & Caballero et al. (2005) \\
\hline 2003 Oct. 22 & 52935.201 & $1 \times 600$ & $<1$ & $3.5 \mathrm{~m} \mathrm{CA}$ & $\mathrm{o} 2 \mathrm{~K}$ & $H$ & Caballero et al. (2005) \\
\hline 2003 Dec. 22 & 52995.596 & $3 \times 600$ & $<1$ & TCS & CAIN-2 & $J$ & this paper \\
\hline 2003 Dec. 22 & 52996.086 & $1 \times 1200$ & $<1$ & TCS & CAIN-2 & $H$ & this paper \\
\hline 2003 Dec. 26 & 53000.092 & $3 \times 120$ & $<1$ & IAC-80 & $\mathrm{CCD}$ & $I$ & this paper \\
\hline 2003 Dec. 28 & 53002.080 & $6 \times 600$ & $\sim 1$ & IAC- 80 & $\mathrm{CCD}$ & $I$ & this paper \\
\hline 2004 Jan. 11 & 53016.116 & $10 \times 300$ & $\sim 1$ & OGS & ESACCD & $W^{a}$ & this paper \\
\hline 2004 Jan. 14 & 53019.061 & $20 \times 300$ & 1.9 & OGS & ESACCD & $W^{a}$ & this paper \\
\hline 2004 Jan. 24 & 53028.859 & $13 \times 600$ & 2.2 & IAC-80 & $\mathrm{CCD}$ & $I$ & this paper \\
\hline 2004 Jan. 25 & 53029.843 & $14 \times 600$ & 2.7 & IAC-80 & $\mathrm{CCD}$ & $I$ & this paper \\
\hline 2004 Jan. 30 & 53034.879 & $3 \times 1200$ & $<1$ & IAC-80 & $\mathrm{CCD}$ & $I$ & this paper \\
\hline 2004 Jan. 30 & 53034.854 & $46 \times 300$ & 4.0 & OGS & ESACCD & $W^{a}$ & this paper \\
\hline 2004 Jan. 31 & 53035.887 & $30 \times 300$ & 2.7 & OGS & ESACCD & $W^{a}$ & this paper \\
\hline 2004 Feb. 1 & 53036.833 & $45 \times 300$ & 6.3 & OGS & ESACCD & $W^{a}$ & this paper \\
\hline 2004 Feb. 2 & 53037.875 & $26 \times 300$ & 4.6 & OGS & ESACCD & $W^{a}$ & this paper \\
\hline 2004 Feb. 3 & 53038.948 & $6 \times 600$ & $\sim 1$ & IAC-80 & $\mathrm{CCD}$ & $I$ & this paper \\
\hline
\end{tabular}

${ }^{a} W$ : optical images taken with no filter.

during the observations. The combined final image is depicted in Fig. 1. The photometric calibration of these data has been taken from Béjar et al. (2004).

Figure 5 portrays the $I$-band light curve of S Ori J053825.4-024241 and of one reference star. Error bars are of the same size as the symbols in the plots. The data with the longest temporal coverage are enlarged in Fig. 6. We have plotted the standard deviation of the light curves of 97 sources in the field of view as a function of $I$-band magnitude in Fig. 7. S Ori J053825.4-024241 stands out from the trend defined by the great majority of the sources ${ }^{1}$. S Ori J053825.4-024241 shows $I$-band variations of $\sim 0.05$ and $\sim 0.25 \mathrm{mag}$ in timescales of hours and days, respectively. We note the marked brightness decrease around MJD $=53035$, which lasted for about one week.

\footnotetext{
1 The most variable objects in this diagram are two sources that are probably background large-amplitude eclipsing binaries that do not belong to the cluster according to their $B R I J H K_{\mathrm{s}}$ magnitudes. One of these binaries shows a light curve with primary and secondary minima of similar amplitude $(\approx 0.6 \mathrm{mag})$ and a period of $\approx 5.5 \mathrm{~h}$ (coordinates J2000: 0538 19.50 -02 41 22.5).
}
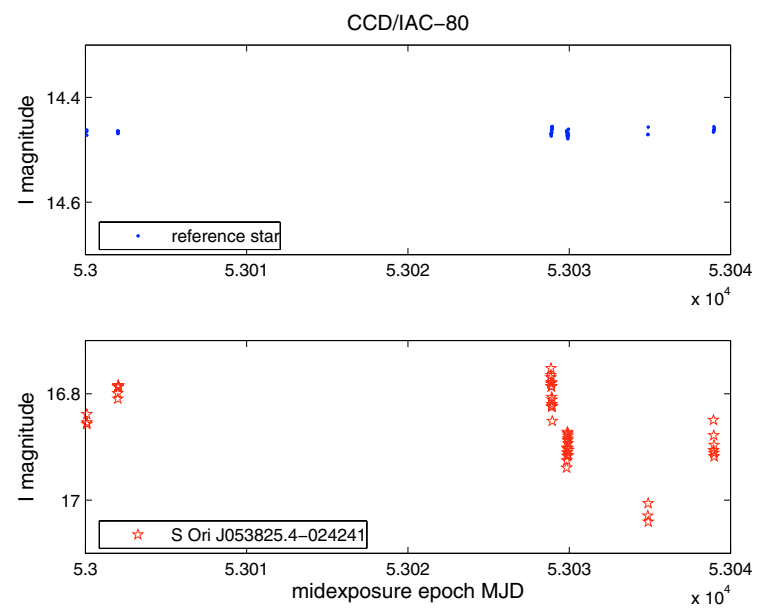

Fig. 5. IAC-80 I-band light curves of S Ori J053825.4-024241 (bottom panel) and of a nearby reference star (top panel). Data have been obtained from Dec. 2003 through Feb. 2004. Note that the vertical scale is the same for the two panels.

\subsubsection{White light differential photometry}

We have used the OGS telescope to photometrically monitor a large area $\left(\sim 0.5 \mathrm{deg}^{2}\right)$ of the $\sigma$ Orionis cluster. The mosaic 

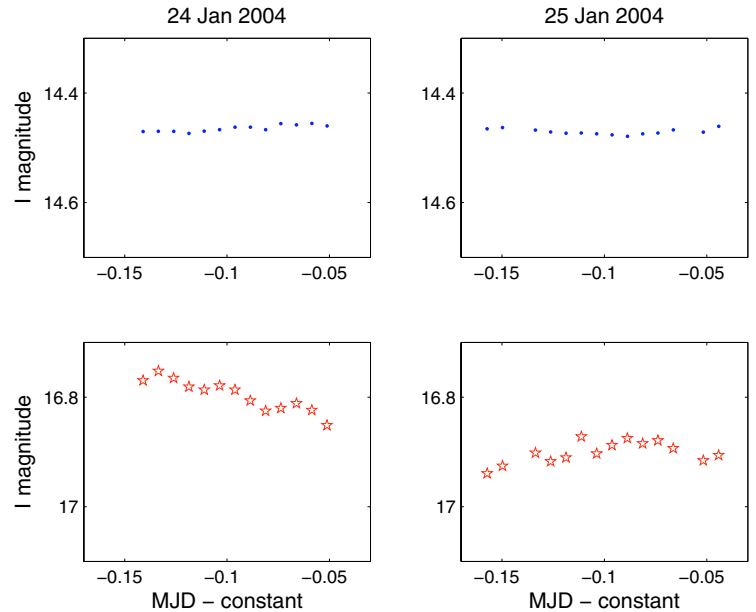

Fig. 6. Details of the IAC-80 I-band light-curves of S Ori J053825.4024241 (bottom panels) and of a nearby reference star (top panels). Our target was monitored for about $2.5 \mathrm{~h}$ during MJD $~ 53029$ (left panels) and 53030 (right panels). It appears that a modulation of lower amplitude and shorter time scale is superimposed on a large photometric variability.

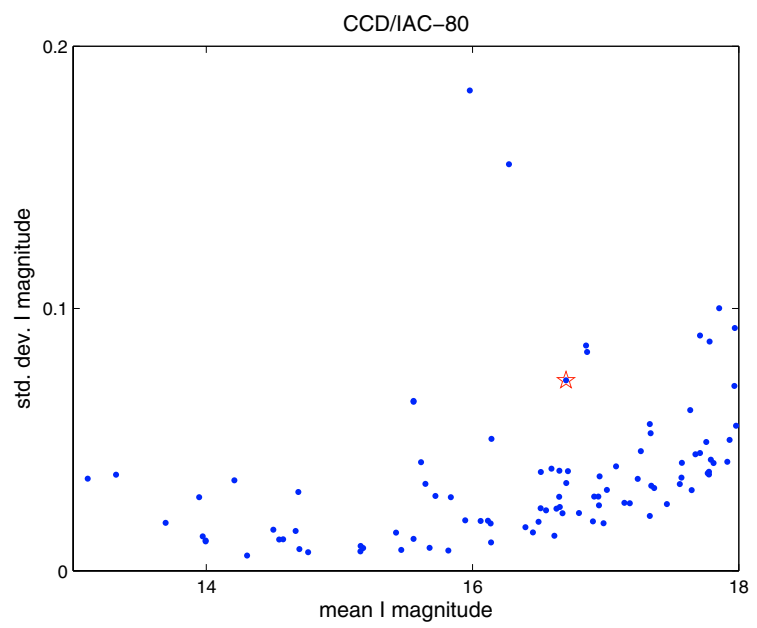

Fig. 7. Standard deviation of the IAC- 80 I-band differential photometry plotted against the average $I$ magnitude. The location of S Ori J053825.4-024241 is marked with a star symbol.

detector of the ESACCD camera comprises four E2V Technologies CCD42-40 devices $(2048 \times 2048$ pixels, 0.62 arcsec pixel $^{-1}$ ), which provides a total field of view of about $0.5 \mathrm{deg}^{2}$ in a single spot. We collected data from January 2004 through to the beginning of February 2004 on six different nights. Here we will focus on the analysis of S Ori J053825.4-024241.

We note that no filter was used when collecting the OGS data. This is indicated by $W$ (for "white light") in the filter column of Table 5. Nevertheless, the detector is sensitive to optical wavelengths. We have computed the effective wavelength of the OGS observations for the case of S Ori J053825.4-024241 by convolving the transmission function of the telescope, the response of the E2V CCDs and the spectral energy distribution of an M6-type dwarf (a significant fraction of the energy is released at red wavelengths). Our

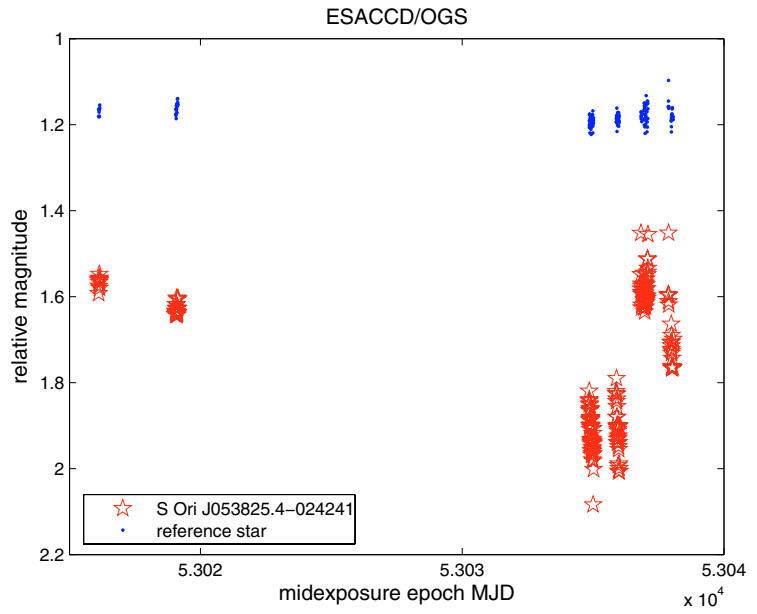

Fig. 8. OGS optical light curve of S Ori J053825.4-024241 (stars) and of a reference star (dots). Data have been obtained from Jan. to Feb. 2004.

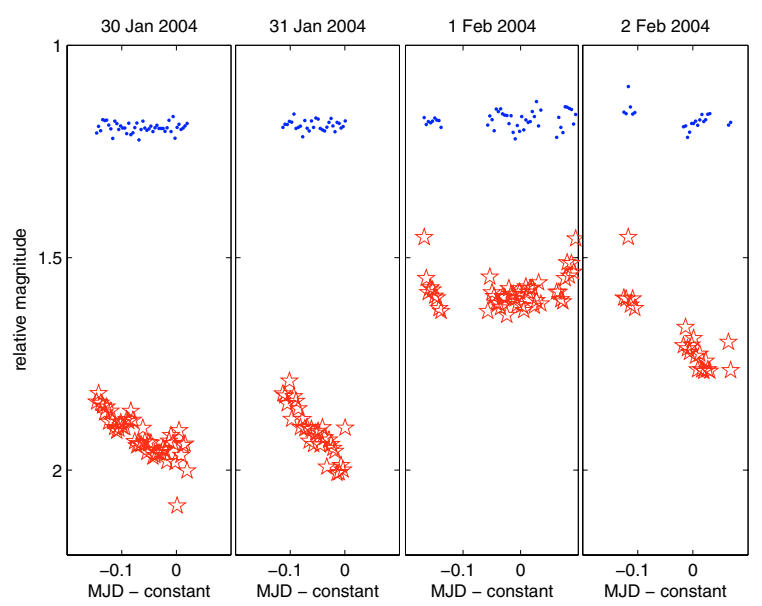

Fig. 9. Details of the OGS light-curve of S Ori J053825.4-024241 (stars) and of a reference star (dots). Our target was monitored for $3-6 \mathrm{~h}$ on each of these four observing nights.

computations yield an effective wavelength of $7800 \AA$, which is intermediate between the Johnson $R(6410 \AA)$ and $I$ bands (8500 $\AA$ ) used at the IAC-80 telescope.

A total of 173 OGS images have been used to construct the light curve of our target. We have not attempted to correct the light curve for colour-dependent differential airmass extinction, as no particular trend is observed between various sources and S Ori J053825.4-024241 from night to night. The minimum variation amplitude detectable from the OGS data is 0.020 mag. Figures $8-10$ show the complete OGS light curve of S Ori J053825.4-024241, the details of four observing nights, and the standard deviation of the light curves as a function of magnitude, respectively. S Ori J053825.4-024241 shows a marked variation amplitude at wavelengths shorter than $8000 \AA$ : $\sim 0.2 \mathrm{mag}$ on timescales of hours, and $0.5 \mathrm{mag}$ from night to night. The brightness decrease at around MJD = 53035 is also observed in the OGS data. 


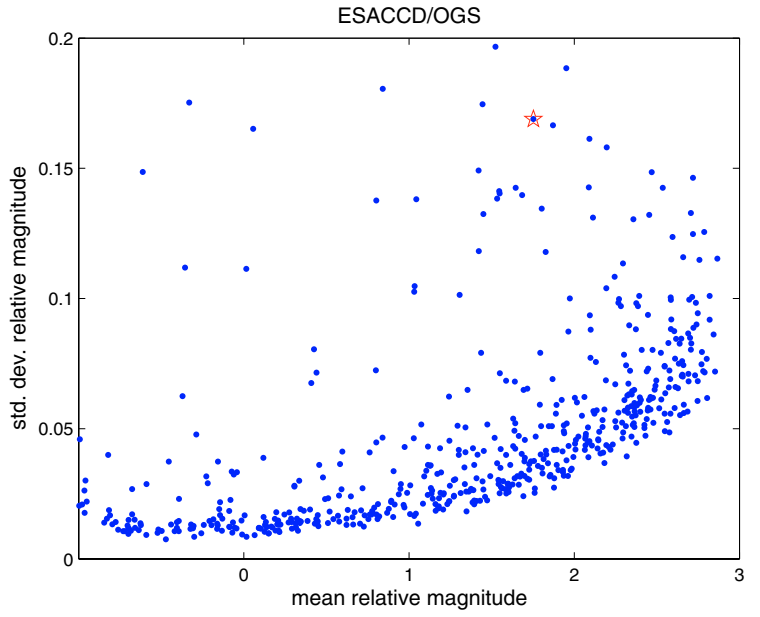

Fig. 10. Standard deviation of the OGS differential photometry plotted against the average relative magnitude. The location of S Ori J053825.4-024241 is marked with a star symbol.

\subsection{Near-infrared photometry}

We have obtained further near-infrared data ( $J$ and $H$ bands) at different epochs. All observations are summarized in Table 5. The instruments used were the NICMOS-3256 × 256 CAIN-2 camera $\left(1.00 \operatorname{arcsec}_{\text {pixel }}^{-1}\right)$ at the TCS telescope, OMEGA2000 at the 3.5 m CAHA telescope and ISAAC at the UT1 Antu. The $J$-band photometry obtained with the TCS in Jan. 1999 was published in Béjar et al. (2004). The remaining nearinfrared photometry of Table 5 is unpublished and presented in this paper for the first time (see Table 6). All near-infrared photometry has been referred to the 2MASS photometric system (S Ori J053825.4-024241 was detected by 2MASS at $\mathrm{MJD}=51116.323$ ). We have carried out $J$ - and $H$-band differential photometry of S Ori J053825.4-024241 with respect to a certain number of reference stars within a radius of $20 \mathrm{arcmin}$ from our target. The minimum variation amplitudes that can be detected are $0.03 \mathrm{mag}$ in $J$ and $0.06 \mathrm{mag}$ in $H$. Our target has been observed in $K_{\mathrm{s}}$ only once by $2 \mathrm{MASS}$. Further discussion on the near-infrared variability of S Ori J053825.4-024241 will be given in Sect. 3.5.

\section{Discussion}

\subsection{Mass estimate}

Stauffer et al. (1998) and Martín et al. (1998) determined that the substellar mass limit lies at spectral type M6 in the Pleiades cluster $(\sim 120 \mathrm{Myr})$. State-of-the-art evolutionary models predict that objects at the substellar boundary evolve at a roughly constant effective temperature (within $200 \mathrm{~K}$ ) between a few million years and the age of the Pleiades to cool down very fast afterwards. Thus, it has been suggested that the star-brown dwarf boundary occurs at M5-6 in $\sigma$ Orionis. The spectral type of S Ori J053825.4-024241 is M 6, i.e. close to the cluster substellar limit. To constrain its mass with greater precision we compare the object luminosity to theoretical predictions. The luminosity of S Ori J053825.4-024241 is derived from its $H$ band average magnitude $(H=14.18 \pm 0.06)$ because, as we
Table 6. Photometric data of S Ori J053825.4-024241.

\begin{tabular}{lcr}
\hline \hline Filter & Magnitude & Source \\
\hline$B^{a}$ & $\sim 20.9$ & USNO-B1 \\
$R^{a}$ & $18.66 \pm 0.05$ & Béjar et al. (2004) \\
$I$ & $16.82-17.52$ & this paper \\
$J$ & $14.57-15.04$ & this paper \\
$H$ & $14.18 \pm 0.06$ & this paper \\
$K_{\mathrm{s}}^{a}$ & $13.57 \pm 0.03$ & 2MASS \\
\hline
\end{tabular}

${ }^{a}$ A single measurement.

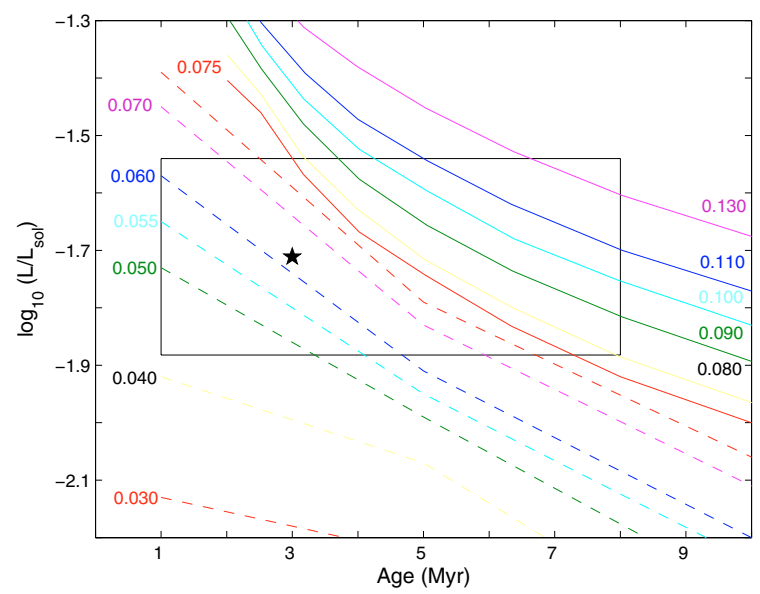

Fig. 11. Models of luminosity evolution of low-mass stars and brown dwarfs from the Lyon group; full lines correspond to NEXTGEN models (Baraffe et al. 1998) and dashed lines to DUSTY models (Chabrier et al. 2000). The most likely location of S Ori J053825.4-024241 is indicated by a star symbol. The box accounts for the uncertainty in the luminosity determination and the probable age of the cluster (1-8 Myr). For ages below 5 Myr, S Ori J053825.4-024241 is very likely substellar.

discuss in Sect. 3.5, this object shows the lowest photometric variability at these wavelengths. In addition, there is no obvious excess emission at $H$ in our data. To transform this colour magnitude into bolometric magnitude, we have used the bolometric correction vs. spectral type relation of Golimowski et al. (2004) and a distance of $360 \pm 70 \mathrm{pc}$ to the $\sigma$ Orionis cluster, deriving $M_{\text {bol }}=9.0 \pm 0.4$. The large error bar associated with this measurement is due to our poor knowledge of the cluster distance.

We show in Fig. 11 the luminosity evolution of objects with masses between 0.03 and $0.13 M_{\odot}$ as provided by the Lyon models (Baraffe et al. 1998, 2003; Chabrier et al. 2000). The most likely location of S Ori J053825.4-024241 in this diagram is indicated by a star symbol at the age of $3 \mathrm{Myr}$, the most probable age of the cluster. The box around this location accounts for the uncertainty in the luminosity determination and the possible age interval (1-8Myr) of the $\sigma$ Orionis cluster. From Fig. 11, the mass of S Ori J053825.4-024241 is estimated at $0.06_{-0.02}^{+0.07} M_{\odot}$, indicating that this object is probably substellar. 


\section{2. $\mathrm{H} \alpha$ emission}

With a $p E W$ of about $-250 \AA$, S Ori J053825.4-024241 is one of the strongest $\mathrm{H} \alpha$ emitters in the $\sigma$ Orionis cluster. The $\mathrm{H} \alpha$ strength of our target is about five times larger than that of the most active low-mass stars of the cluster, while only two substellar objects, S Ori 55 and 71, show higher $\mathrm{H} \alpha$ emission (Zapatero Osorio et al. 2002b; Barrado y Navascués et al. 2002). The marked persistence of the $\mathrm{H} \alpha$ line of S Ori J053825.4-024241, which is always seen in substantial emission in our data, suggests that its origin is not due to a transient event. As mentioned in Sect. 2.1, S Ori J053825.4-024241 shows broad $\mathrm{H} \alpha$ wings (Fig. 3). After correction for the contribution of the instrumental profile, the real $\mathrm{H} \alpha$ FWHM turns out to be $230 \mathrm{~km} \mathrm{~s}^{-1}$ (or $500 \mathrm{~km} \mathrm{~s}^{-1}$ if we consider the pedestal of the line at the $10 \%$ level of the peak flux). Such large velocity appears to be close to the breakup limit of S Ori J053825.4-024241, so the $\mathrm{H} \alpha$ broadening is probably not caused by intrinsic rotation. We interpret the persistent broad $\mathrm{H} \alpha$ emission as due to hot mass infall or mass ejection (e.g., White \& Basri 2003).

To compare the relative strength of the $\mathrm{H} \alpha$ emission of S Ori J053825.4-024241 with those of other low-mass $\sigma$ Orionis members, we have evaluated the $L_{\mathrm{H} \alpha} / L_{\mathrm{bol}}$ ratio of our target. This quantity is independent of the surface area and represents the fraction of the total energy output in $\mathrm{H} \alpha$. The bolometric luminosity was calculated using the $M_{\text {bol }}$ given in Sect. 3.1 and the solar bolometric magnitude of 4.74. We followed Hodgkin et al. (1995) for obtaining $L_{\mathrm{H} \alpha}$ from the width of the line, the $(R-I)_{\mathrm{C}}$ colour and the $V$-band magnitude. The $(R-I)_{\mathrm{C}}$ colour is taken from Béjar et al. (2004), who provide quasi-simultaneous photometry in the two bands. We have estimated $V$ to be $21.0 \pm 0.5 \mathrm{mag}$ by adopting the $(V-I)_{C}$-spectral type relationship of Kirkpatrick et al. (1994). The NOT and Keck $\mathrm{H} \alpha$ measurements yield similar values within the error bar, i.e. $\log \left(L_{\mathrm{H} \alpha} / L_{\mathrm{bol}}\right)=-2.4 \pm 0.3^{2}$. The error bar takes into account photometric uncertainties and the error in the distance to the cluster. Figure 12 shows $\log \left(L_{\mathrm{H} \alpha} / L_{\mathrm{bol}}\right)$ as a function of spectral type for cluster members. Except for our target, data have been gathered from Zapatero Osorio et al. (2002a,b) and Barrado y Navascués et al. (2002). S Ori J053825.4-024241 exhibits quite intense emission, which is comparable to that of active stellar accretors.

\subsection{Other emission lines and radial velocity}

The "photospheric" radial velocity of S Ori J053825.4-024241 $\left(v_{\mathrm{h}}=+32 \pm 13 \mathrm{~km} \mathrm{~s}^{-1}\right)$ is consistent with membership of the $\sigma$ Orionis cluster. The cluster systemic velocity is in the range 27 to $38 \mathrm{~km} \mathrm{~s}^{-1}$ (Bohannan \& Garmany 1978; Garmany et al. 1980; Morrell \& Levato 1991). Zapatero Osorio et al. (2002a) and Kenyon et al. (2005) found mean heliocentric velocities of $37 \pm 6$ and $31.2 \pm 0.2 \mathrm{~km} \mathrm{~s}^{-1}$, respectively, for lowmass members of the cluster.

\footnotetext{
${ }^{2}$ If the object were brighter in $V$ than expected, e.g. $V=20.0 \pm 0.5$, $\log \left(L_{\mathrm{H} \alpha} / L_{\mathrm{bol}}\right)$ could be as high as $-2.0 \pm 0.3$.
}

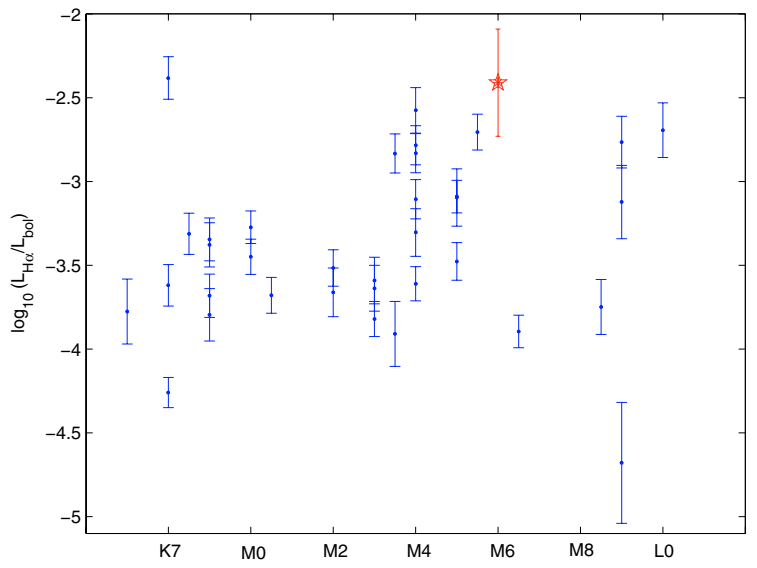

Fig. 12. H $\alpha$ luminosity over bolometric luminosity is plotted against spectral type for $\sigma$ Orionis members. S Ori J053825.4-024241 (star symbol) exhibits a significant amount of $\mathrm{H} \alpha$ emission in comparison to other cluster low-mass stars and brown dwarfs.

Emission lines, if originating outside the photosphere, may show a different velocity. The measurements of $\mathrm{H} \alpha$ and He I $\lambda 5876$ are in agreement with the "photospheric" velocity of S Ori J053825.4-024241 within the $1 \sigma$ uncertainty. However, [O I] $\lambda 6300$ shows a moderate blue-shifted velocity of about $2 \sigma$. This may indicate the presence of low density gas outflow (mass loss, wind), which is a process commonly found in Classical T Tauri stars with jets. The red component of this emission line might be hidden by an opaque disc (Appenzeller et al. 1984; Edwards et al. 1987). It has also been suggested (Corcoran \& Ray 1997) that low velocity forbidden line emission (like the one we have measured in S Ori J053825.4-024241) could be due to disc wind.

\subsection{Lithium and optical veiling}

Lithium at $\lambda 6707.8 \AA$ is not detected in any of our spectroscopic data. From the observed spectra, we can impose upper limits on the line $p E W$ of $+0.7 \AA$ (NOT) and $+0.3 \AA$ (Keck). Because of the very young age of $\sigma$ Orionis, it is expected that all cluster stars and brown dwarfs have depleted none of their initial lithium abundance.

We have computed a few synthetic spectra around lithium using the code of Pavlenko et al. (2000), and $T_{\text {eff }}=2900 \mathrm{~K}$, $\log g=4.0$ and solar metallicity, which are the expected photospheric parameters of an M6-type dwarf in $\sigma$ Orionis. The theoretical spectra have been degraded to match the resolution of $4 \AA$. Computations have been carried out considering two different lithium abundances $(\log N(\mathrm{Li})=3.0$, i.e. no depletion, and complete depletion), and the presence of veiling. S Ori J053825.4-024241 shows a very strong $\mathrm{H} \alpha$ emission suggestive of intense accretion; furthermore, the $R-I$ colour and the titanium oxide bands of this object appear bluer and weaker, respectively, than expected for M6 dwarfs, possibly indicating the presence of some blue continuum excess. For our computations, we have adopted a flat energy distribution in the wavelength interval 6672-6740 for the flux continuum excess. Our results are depicted in Fig. 13, where the top panel 

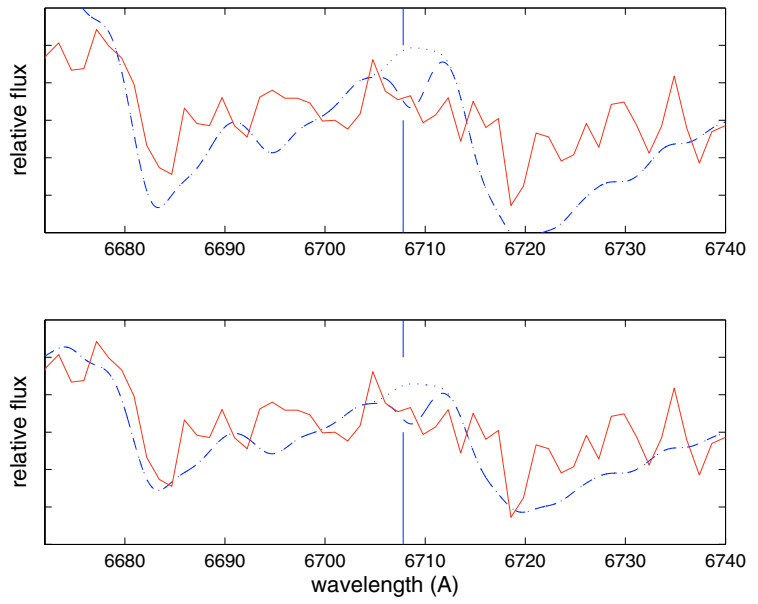

Fig. 13. The Keck spectrum of S Ori J053825.4-024241 (solid line) is compared to "unveiled" synthetic spectra computed for $\log N(\mathrm{Li})=$ 3.0 (dashed line) and no lithium (dotted line) in the top panel. The bottom panel shows the comparison between S Ori J053825.4-024241 (solid line) and a "veiled" $(r=0.5)$ theoretical spectrum with $\log N(\mathrm{Li})=3.0$ (dashed line). The location of the lithium feature is indicated by a vertical line.

shows the comparison between the observed Keck spectrum of S Ori J053825.4-024241 and the "unveiled" synthetic spectra, and the bottom panel displays S Ori J053825.4-024241 and the "veiled" computations. It is apparent from the figure that the TiO features of S Ori J053825.4-024241 are better reproduced by the spectral synthesis including some veling. An amount of $r=0.5$, where $r=F_{\text {excess }} / F_{\text {phot }}$, reasonably matches the observations. The lithium absorption feature is detectable in the unveiled, lithium-rich, $4 \AA$ A-resolution theoretical spectrum $(p E W \approx 0.3 \AA)$, whereas it becomes rather weak in the veiled spectrum. Therefore, the non-detection of lithium in our data is still consistent with complete preservation. Higher-resolution spectra are needed to study lithium in S Ori J053825.4-024241.

\subsection{Photometric variability and the Classical T Tauri scenario}

Based on our optical data, we conclude that S Ori J053825.4024241 presents the largest variability among very low-mass stars and brown dwarfs so far identified in the $\sigma$ Orionis cluster The amplitude of the variations in the $I$-band $(8500 \AA)$ are $\sim 0.05 \mathrm{mag}$ in timescales of hours and $\sim 0.25 \mathrm{mag}$ on timescales of days. The variability is even larger at shorter wavelengths. These conclusions strongly support the Classical T Tauri (CTT) picture. As noted by Appenzeller \& Mundt (1989), all carefully monitored CTT stars have turned out to be variables. We have searched for a periodic signal in the IAC80 and OGS data of S Ori J053825.4-024241 by calculating the power spectrum as described in Caballero et al. (2004). We have found no significant peaks in the periodograms.

To study the long-term optical photometric variability of S Ori J053825.4-024241 we have compared our data to previously published data obtained at different epochs and using similar instrumentation. These data and the new
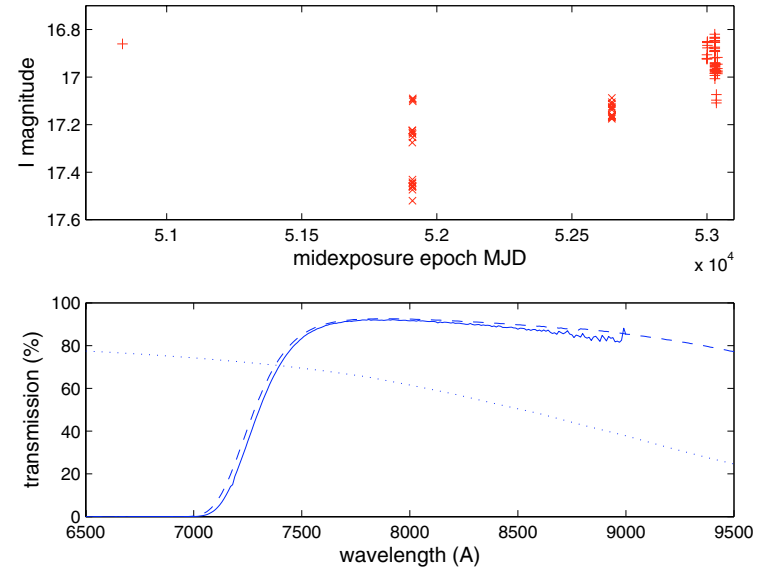

Fig. 14. The long-term I-band light-curve of S Ori J053826.1-024041 is depicted in the top panel. Data, which include the INT (crosses) and IAC- 80 (pluses) photometry, span a time coverage of six years. The bottom panel displays the transmission curves of the IAC-80 (dashed line) and the INT (RGO-I, full line) I-band filters and of one of the CCDs (dotted line) of the INT camera.

photometry presented here span a total of 6 years of observations. The $I$-band filters used at the IAC- 80 and INT are very similar as inferred from their transmission curves shown in the bottom panel of Fig. 14. For direct comparison, all data have been referred to the same photometric calibration as in Béjar et al. (2004). The comparison of the light curves of the reference stars in common between the IAC- 80 and INT datasets yields a dispersion of 0.025 mag. The complete set of $I$-band observations is depicted in the top panel of Fig. 14. S Ori J053825.4-024241 also presents long-term photometric varibility in the optical with an amplitude of $0.7 \mathrm{mag}$. This is larger than the short-term variation amplitude. Further data are needed to investigate whether the optical light curve of S Ori J053825.4-024241 consists of a long, very large amplitude variation on to which variations of smaller amplitudes and shorter timescales are superimposed (see Scholz \& Eislöffel 2004). We provide in Table 6 the intervals of the photometric magnitudes that we have measured in the optical and near-infrared.

Figure 15 shows all near-infrared photometry of S Ori J053825.4-024241 available to date as a function of the epoch of the observations. The $H$-band photometry appears to be rather stable within $1 \sigma$ the uncertainty. At $J$, our target shows a large scatter, suggesting variability. The amplitude of the variations is about $0.5 \mathrm{mag}$, which compares with the optical variability. The photometric variability of CTT stars decreases towards longer wavelengths because the origin of the variations probably lie in hot and/or cold photospheric spots covering a significant percentage of the object surface. It is also believed that part of the variability is due to occultations by inhomogeneities of the inner disc regions, which show less dependence on wavelength. This picture might be applicable to S Ori J053825.4-024241 to explain its peculiar optical and near-infrared light-curves. We note that S Ori J053825.4-024241 presents a clear flux excess 


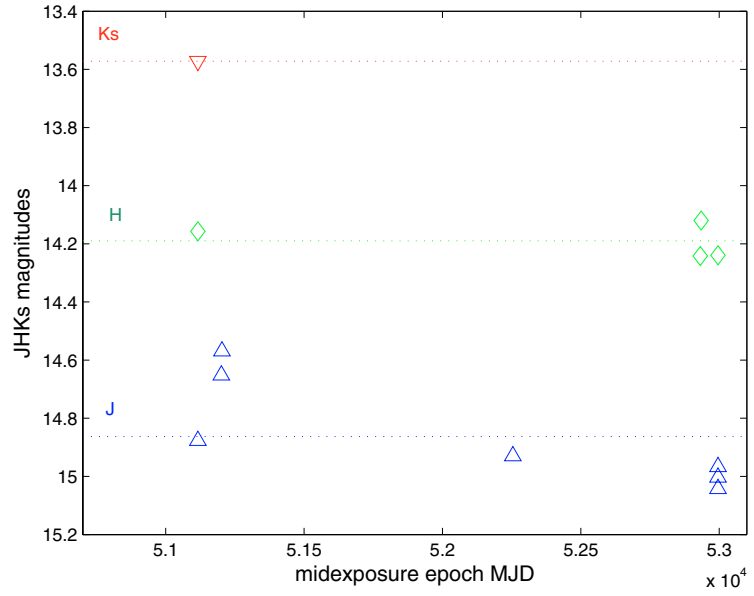

Fig. 15. $J H K_{\mathrm{s}}$-band light-curves of S Ori J053825.4-024241 ( $\mathrm{J}: \triangle ; H$ : $\left.\diamond ; K_{\mathrm{s}}: \nabla\right)$. The dotted lines stand for the average magnitude in each filter.

at $2.2 \mu \mathrm{m}$ (Béjar et al. 2004; Caballero et al. 2004), which may be associated with the presence of a surrounding disc.

Interestingly, S Ori J053825.4-024241 is detected in the USNO-B1 blue plates (Monet et al. 2003), from which we have obtained the blue photographic magnitude. This magnitude has been converted into the standard $B$ magnitude by applying the conversion equation of Zombeck (1990). We provide our measurement in Table 6 . From the $B$ magnitude and the typical colours of M5-7 dwarfs, it is also apparent that S Ori J053825.4-024241 presents a flux excess at blue wavelengths. This result is consistent with the presence of veiling as discussed in the previous section. We note that on some occasions, when the accretion activity is large, S Ori J053825.4-024241 may show up bluer in the optical than other cluster members of similar types. This might explain why this object was not selected as a cluster member candidate from previous $R I$ surveys in the region.

\section{Summary and conclusions}

We have obtained low-resolution optical spectroscopy $(R \sim$ 600 ) in the wavelength interval $5300-9600 \AA$ of four lowmass candidate members of the $\sigma$ Orionis cluster $(360 \pm 70 \mathrm{pc}$, $1-8 \mathrm{Myr}$ ). These objects were selected from the photometric surveys by Béjar et al. (2004) and Caballero et al. (2004). We have determined spectral types between M5 and M6 for these sources, which are consistent with our expectations for true cluster members. Furthermore, all of them show spectroscopic features typical of low-gravity atmospheres, supporting their membership of the cluster.

One object, namely S Ori J053825.4-024241 (M 6.0 1.0 ), was known to be variable in the $I$ band (Caballero et al. 2004) and to show slightly bluer $R-I$ and redder $J-K_{\mathrm{s}}$ colours than other $\sigma$ Orionis members of similar magnitude (Béjar et al. 2004). From our low-resolution spectroscopic data, S Ori J053825.4-024241 presents a strong $\mathrm{H} \alpha$ emission (one of the largest among known low-mass cluster members), which, in addition to the near-infrared flux excess, suggests that this object is undergoing accretion processes from a surrounding disc.

To confirm the possible T Tauri nature of S Ori J053825.4024241, we have obtained follow-up higher resolution spectra $(R \sim 1700)$ in the wavelength interval 5600-8100 and have photometrically monitored the object in the $I, J$ and $H$ bands and in white light. S Ori J053825.4-024241 shows persistent, strong $\mathrm{H} \alpha$ emission. In addition, other forbidden and permitted emission lines have been detected, e.g. [OI] $\lambda 6300.3$ and He I 75875.8 . The pseudo-equivalent widths of all these emission lines are consistent with the measurements of known stellar accretors. Furthermore, $\mathrm{H} \alpha$ is found to be broadened with widths typical of $\mathrm{T}$ Tauri stars, which indicates the presence of gas infall at high velocities. From the comparison of our spectroscopic data to theoretical spectra, we infer that some continuum flux excess is present at short wavelengths. We quantify this "veiling" to be around 0.5 in the Li I $\lambda 6707.8$ region. S Ori J053825.4-024241 has a radial velocity consistent with membership of the $\sigma$ Orionis cluster.

S Ori J053825.4-024241 is confirmed to be an irregular variable object. Variations, which do not show any obvious modulation pattern, are significant $(0.05-0.7 \mathrm{mag})$ from blue wavelengths up to the $J$ band. Variability is found at all timescales. Our $H$-band photometry appears rather stable in time, suggesting little variability at wavelengths greater than $1.2 \mu \mathrm{m}$. Both the photometric and spectroscopic properties of S Ori J053825.4-024241 are consistent with the T Tauri scenario. Assuming that S Ori J053825.4-024241 is a member of the $\sigma$ Orionis cluster, we have estimated its mass at $0.06_{-0.02}^{+0.07} M_{\odot}$ after comparison with state-of-the-art evolutionary models. Based on our data, we conclude that S Ori J053825.4-024241 is probably a brown dwarf undergoing processes similar to those of $\mathrm{T}$ Tauri stars.

Acknowledgements. We would like to thank Terry Mahoney for revising the English of the manuscript and the anonymous referee for comments and suggestions that improved the article. J.A.C. thanks to staff at the Observatorio del Teide for performing the Observaciones de Tiempo de Servicio and to David Barrado y Navascués, Gabriel Bihain, Jorge Sanz Forcada and María del Mar Sierra for helpful comments. Partial financial support was provided by the Spanish Ministerio de Ciencia y Tecnología, project AYA20011657 of the Plan Nacional de Astronomía y Astrofísica, and Ministerio de Educación y Ciencia, projects AYA2001-1657 and AYA200305355. YP's studies are partially supported by Royal Society and Leverhulme Trust grants. Research presented herein was partially funded by NSF research grant AST 02-05862. Acknowledgements of the use of telescopes, instruments, catalogues and software can be found in the electronic version.

\section{References}

Appenzeller, I., \& Mundt, R. 1989, A\&ARv, 1, 291

Appenzeller, I., Jankovics, I., \& Östreicher, R. 1984, A\&A, 141, 108

Baraffe, I., Chabrier, G., Allard, F., \& Hauschildt, P. H. 1998, A\&A, 337,403

Baraffe, I., Chabrier, G., Barman, T., Allard, F., \& Hauschildt, P. H. 2003, A\&A, 402, 701

Barrado y Navascués, D., Zapatero Osorio, M. R., Martín, E. L., et al. 2002, A\&A, 393, L85 
Barrado y Navascués, D., \& Martín, E. L. 2003, AJ, 126, 2997

Barrado y Navascués, D., Béjar, V. J. S., Mundt, R., et al. 2003, A\&A, 404, 171

Béjar, V. J. S., Martín, E. L., Zapatero Osorio, M. R., et al. 2001, ApJ, 556,830

Béjar, V. J. S., Zapatero Osorio, M. R., \& Rebolo, R. 2004, AN, 325, 705

Bertout, C., Basri, G., \& Bouvier, J. 1988, ApJ, 330, 350

Bohannan, B., \& Garmany, C. D. 1978, ApJ, 223, 908

Caballero, J. A., Béjar, V. J. S., Rebolo, R., \& Zapatero Osorio, M. R. 2004, A\&A, 424, 857

Caballero, J. A. on behalf of the JOvIAN Collaboration 2005, IAC/TNG Workshop on Ultralow-mass star formation and evolution, La Palma, 2005 June 28-July 1, ed. E. Martín, \& A. Magazzù, in press

Chabrier, G., Baraffe, I., Allard, F., \& Hauschildt, P. 2000, 542, 464

Corcoran, M., \& Ray, T. P. 1997, A\&A, 321, 189

Edwards, S. E., Cabrit, S., Strom, S. E., et al. 1987, ApJ, 321, 473

Furlan, E., Calvet, N., D’Alessio, P., et al. 2005, ApJ, 621, L129

Garmany, C. D., Conti, P. S., \& Massey, P. 1980, ApJ, 242, 1063

Golimowski, D. A., Leggett, S. K., Marley, M. S., et al. 2004, AJ, 127, 3516

Hodgkin, S. T., Jameson, R. F., \& Steele, I. A. 1995, MNRAS, 274, 869

Kenyon, M. J., Jeffries, R. D., Naylor, T., Oliveira, J. M., \& Maxted, P. F. L. 2005, MNRAS, 356, 89

Kirkpatrick, J. D., McGraw, J. T., Hess, T. R., Liebert, J., \& McCarthy, D. W., Jr. 1994, ApJS, 94, 749

Königl, A. 1991, ApJ, 370, L39
Luhman, K. L., Liebert, J., \& Rieke, G. H. 1997, ApJ, 489, L165

Martín, E. L., Rebolo, R., \& Zapatero Osorio, M. R. 1996, ApJ, 469, 706

Martín, E. L., Basri, G., Gallegos, J. E., et al. 1998, ApJ, 499, L61

Mohanty, S., Jayawardhana, R., \& Basri, G. 2005, ApJ, 626, 498

Monet, D. G., Levine, S. E., Casian, B., et al. 2003, AJ, 125, 984

Morrell, N., \& Levato, H. 1991, ApJS, 75, 965

Muzerolle, J., Hillenbrand, L., Calvet, N., Briceño, C., \& Hartmann, L. 2003, ApJ, 592, 266

Natta, A., Testi, L., Muzerolle, J., et al. 2004, A\&A, 424, 603

Oliveira, J. M., Jeffries, J. D., \& van Loon, J. Th. 2004, MNRAS, 347, 1327

Osterbrock, D. E., Fulbright, J. P., Martel, A. R., et al. 1996, PASP, 108, 277

Pavlenko, Ya., Zapatero Osorio, M. R., \& Rebolo, R. 2000, A\&A, 354, 229

Scholz, A., \& Eislöffel, J. 2004, A\&A, 419, 249

Stauffer, J. R., Schultz, G., \& Kirkpatrick, J. D. 1998, ApJ, 499, L199

Tinney, C. G., \& Reid, I. N. 1998, MNRAS, 301, 1031

Walter, F. M., Wolk, S. J., \& Sherry, W. 1998, ASP Conf. Ser., 154, CD-1793

White, R. J., \& Basri, G. 2003, ApJ, 582, 1109

Zapatero Osorio, M. R., Béjar, V. J. S., Pavlenko, Ya., et al. 2002a, A\&A, 384, 937

Zapatero Osorio, M. R., Béjar, V. J. S., Martín, E. L., Barrado y Navascués, D., \& Rebolo, R. 2002b, ApJ, 569, L99

Zombeck, M. V. 1990, Handbook of Astronomy and Astrophysics, Second Edition (Cambridge, UK: Cambridge University Press) 


\section{Online Material}


Acknowledgements. The Telescopio IAC-80 and the Telescopio Carlos Sánchez are operated on the island of Tenerife by the Instituto de Astrofísica de Canarias in the Spanish Observatorio del Teide of the Instituto de Astrofísica de Canarias. Based on observations made with the European Space Agency Orbital Ground Station telescope operated on the island of Tenerife by the Instituto de Astrofísica de Canarias in the Spanish Observatorio del Teide of the Instituto de Astrofísica de Canarias. Based on observations made with the Nordic Optical Telescope, operated on the island of La Palma jointly by Denmark, Finland, Iceland, Norway, and Sweden, in the Spanish Observatorio del Roque de los Muchachos of the Instituto de Astrofísica de Canarias. The data presented here have been taken using ALFOSC, which is owned by the Instituto de Astrofísica de Andalucía (IAA) and operated at the Nordic Optical Telescope under agreement between IAA and the NBIfAFG of the Astronomical Observatory of Copenhagen. Some of the data presented herein were obtained at the W. M. Keck Observatory, which is operated as a scientific partnership among the California Institute of Technology, the University of California and the National Aeronautics and Space Administration. The Observatory was made possible by the generous financial support of the W. M. Keck Foundation. The authors wish to recognize and acknowledge the very significant cultural rôle and reverence that the summit of Mauna Kea has always had within the indigenous Hawaiian community. We were most fortunate to have had the opportunity to conduct observations from this mountain. This research has made use of the SIMBAD database, operated at CDS, Strasbourg, France. This publication makes use of data products from the Two Micron All Sky Survey, which is a joint project of the University of Massachusetts and the Infrared Processing and Analysis Center/California Institute of Technology, funded by the National Aeronautics and Space Administration and the National Science Foundation. The Guide Star Catalogue-II is a joint project of the Space Telescope Science Institute and the Osservatorio Astronomico di Torino. Space Telescope Science Institute is operated by the Association of Universities for Research in Astronomy, for the National Aeronautics and Space Administration under contract NAS5-26555. The participation of the Osservatorio Astronomico di Torino is supported by the Italian Council for Research in Astronomy. Additional support is provided by European Southern Observatory, Space Telescope European Coordinating Facility, the International GEMINI project and the European Space Agency Astrophysics Division. IRAF is distributed by National Optical Astronomy Observatories, which are operated by the Association of Universities for Research in Astronomy, Inc., under cooperative agreement with the National Science Foundation. 